2020, Instituto Mexicano de Tecnología del Agua

Tecnología y

Ciencias $\cong$ Agua

Open Access bajo la licencia CC BY-NC-SA 4.0

(https://creativecommons.org/licenses/by-nc-sa/4.0/)

DOI: $10.24850 /$ j-tyca-2020-02-06

Artículos

\title{
Cambio climático y retroceso glaciar en la Cordillera Huaytapallana, Perú
}

\section{Climate change and glacier retreat in the Huaytapallana Mountain Range, Peru}

Wilfredo Bulege-Gutiérrez ${ }^{1}$, ORCID: 0000-0002-9059-4003

María Custodio², ORCID: 0000-0003-1994-010X

\footnotetext{
${ }^{1}$ Universidad Continental, Huancayo, Perú, wbulege@continental.edu.pe

${ }^{2}$ Universidad Nacional del Centro del Perú, Huancayo, Perú, mcustodio@uncp.edu.pe
}

Autor para correspondencia: Wilfredo Bulege-Gutiérrez, wbulege@continental.edu.pe

\section{Resumen}

El objetivo ha sido describir y determinar la relación de la temperatura ambiental —como manifestación del cambio climático-y el retroceso de la masa glaciar de la Cordillera Huaytapallana en Perú. Los datos de temperatura mínima y superficie glaciar se obtuvieron a partir de 
Tecnología y

Ciencias $\approx$ Agua
2020, Instituto Mexicano de Tecnología del Agua

Open Access bajo la licencia CC BY-NC-SA 4.0

(https://creativecommons.org/licenses/by-nc-sa/4.0/)

informes oficiales del Senamhi e IGP están expresadas en ${ }^{\circ} \mathrm{C}$ y $\mathrm{km}^{2}$, respectivamente. Los datos de temperatura pertenecen al Observatorio de Huancayo, ubicado en latitud $12^{\circ} 02^{\prime} \mathrm{S}$, longitud $75^{\circ} 19^{\prime} \mathrm{W}$, altitud 3 313 msnm en el distrito de Huachac, provincia de Chupaca, departamento de Junín. Los datos de superficie glaciar fueron tomados de estudios de la Cordillera Huaytapallana por el Instituto Geofísico del Perú. La estimación de datos faltantes de las variables se realizó con un modelo de regresión lineal. Entre los resultados se tiene que, entre 1986 y 2016 es evidente un incremento de la temperatura mínima anual en la zona de estudio, mostrando valores entre 3.435 y $5.227^{\circ} \mathrm{C}$; se estima al 2016 un valor de $4.757^{\circ} \mathrm{C}$; asimismo, la superficie de masa glaciar de la Cordillera Huaytapallana muestra en este mismo periodo una tendencia de retroceso, estimado al 2016 en 11.86 km². En conclusión, el incremento de la temperatura mínima del periodo 1986-2016 incide en el retroceso de la superficie de masa glaciar de la Cordillera Huaytapallana; de igual forma, existe una relación inversa, moderada y significativa entre el incremento de la temperatura mínima y el retroceso de la masa glaciar de la cordillera objeto de estudio.

Palabras clave: temperatura mínima, superficie glaciar, Cordillera Huaytapallana.

\section{Abstract}

The objective has been to describe and determine the relationship between environmental temperature -as a manifestation of climate change- and the glacier mass retreat of Huaytapallana mountain range 
Tecnología y

Ciencias
2020, Instituto Mexicano de Tecnología del Agua

Open Access bajo la licencia CC BY-NC-SA 4.0

(https://creativecommons.org/licenses/by-nc-sa/4.0/)

in Peru. The minimum temperature and glacier surface data were obtained from Senamhi and IGP official reports, they are expressed in ${ }^{\circ} \mathrm{C}$ and $\mathrm{km}^{2}$ respectively. The temperature data belong to the Huancayo Observatory located at Latitude $12 \circ 02$ 'S, Longitude $75 \circ 15^{\circ}$ W, Altitude 3313 masl; in the Huachac district, Chupaca province, Junín department. The glacier surface data were taken from the mountain Huaytapallana's studies by the Instituto Geofísico del Perú. The variables' missing data estimation was performed with a linear regression model. Among the results we have found that between 1986 to 2016, an increase in the minimum annual temperature in the study area is evident, showing values between 3.435 to $5.227^{\circ} \mathrm{C}$; a value of $4.757^{\circ} \mathrm{C}$ is estimated at 2016 ; likewise, the glacial mass surface of the Huaytapallana mountain shows a downward trend in this same period, estimated at 2016 in $11.86 \mathrm{~km}^{2}$. In conclusion, the increase of minimum temperature of the period 1986 - 2016 affects the glacier mass surface retreat of the Huaytapallana mountain range; likewise, there is an inverse, moderate and significant relationship between the minimum temperature increase and the glacier mass retreat of the mountain which is our study object.

Keywords: Minimum temperature, glacier surface, Huaytapallana mountain range.

Recibido: 7/11/2017

Aceptado: 23/09/2019 
Tecnología y

Ciencias $\approx$ Agua
2020, Instituto Mexicano de Tecnología del Agua

Open Access bajo la licencia CC BY-NC-SA 4.0

(https://creativecommons.org/licenses/by-nc-sa/4.0/)

\section{Introducción}

El cambio climático global es la variación del estado del clima, identificable ( $p$. ej., mediante pruebas estadísticas) en las variaciones del valor medio o en la variabilidad de sus propiedades, que persiste durante largos periodos de tiempo, generalmente decenios, o periodos más largos. El cambio climático puede deberse a procesos internos naturales o a forzamientos externos, como modulaciones de los ciclos solares, erupciones volcánicas o cambios antrópicos persistentes de la composición de la atmósfera o del uso del suelo (Panel Intergubernamental de Cambio Climático, 2014).

La Convención Marco de las Naciones Unidas sobre el Cambio Climático (CMNUCC), en su artículo 1 , define el cambio climático como "cambio de clima atribuido directa o indirectamente a la actividad humana que altera la composición de la atmósfera global y que se suma a la variabilidad natural del clima observada durante períodos de tiempo comparables". La CMNUCC diferencia, pues, entre el cambio climático atribuible a las actividades humanas que alteran la composición atmosférica y la variabilidad climática atribuible a causas naturales (Panel Intergubernamental de Cambio Climático, Grupo de Trabajo II, 2014). 
Tecnología y

Ciencias $₫$ Agua
2020, Instituto Mexicano de Tecnología del Agua

Open Access bajo la licencia CC BY-NC-SA 4.0

(https://creativecommons.org/licenses/by-nc-sa/4.0/)

De acuerdo con el Panel Intergubernamental de Cambio Climático (2014), el calentamiento en el sistema climático es inequívoco, y desde la década de 1950 muchos de los cambios observados no han tenido precedentes en los últimos decenios a milenios. La atmósfera y el océano se han calentado, los volúmenes de hielo y nieve han disminuido, y el nivel del mar se ha elevado. Cada uno de los tres últimos decenios ha sido sucesivamente más cálido en la superficie de la Tierra que cualquier decenio anterior desde 1850. Es probable que el periodo 1983-2012 haya sido el periodo de treinta años más cálido de los últimos 1400 años en el hemisferio norte, donde es posible realizar esa evaluación (nivel de confianza medio). Los datos de temperatura de la superficie terrestre y oceánica, combinados y promediados en lo global, calculados a partir de una tendencia lineal, muestran un calentamiento de $0.85(0.65-1.06){ }^{\circ} \mathrm{C}$, durante $1880-2012$, para el que se han producido de forma independiente varios conjuntos de datos.

Además de registrar un calentamiento multidecenal notable, la temperatura media global en superficie muestra una variabilidad decenal e interanual considerable (Figura 1). Debido a esa variabilidad natural, las tendencias basadas en periodos de registros cortos son muy sensibles a las fechas de inicio y final, y no reflejan, en general, las tendencias climáticas a largo plazo. Por ejemplo, la tasa de calentamiento durante los últimos 15 años (0.05 [-0.05 a 0.15] ${ }^{\circ} \mathrm{C}$ por decenio, entre 1998 y 2012), que comienza con un fuerte efecto del fenómeno El Niño, es menor que la tasa registrada desde 1951 (0.12 [0.08 a 0.14$]^{\circ} \mathrm{C}$ por decenio, entre 1951 y 2012 ). 


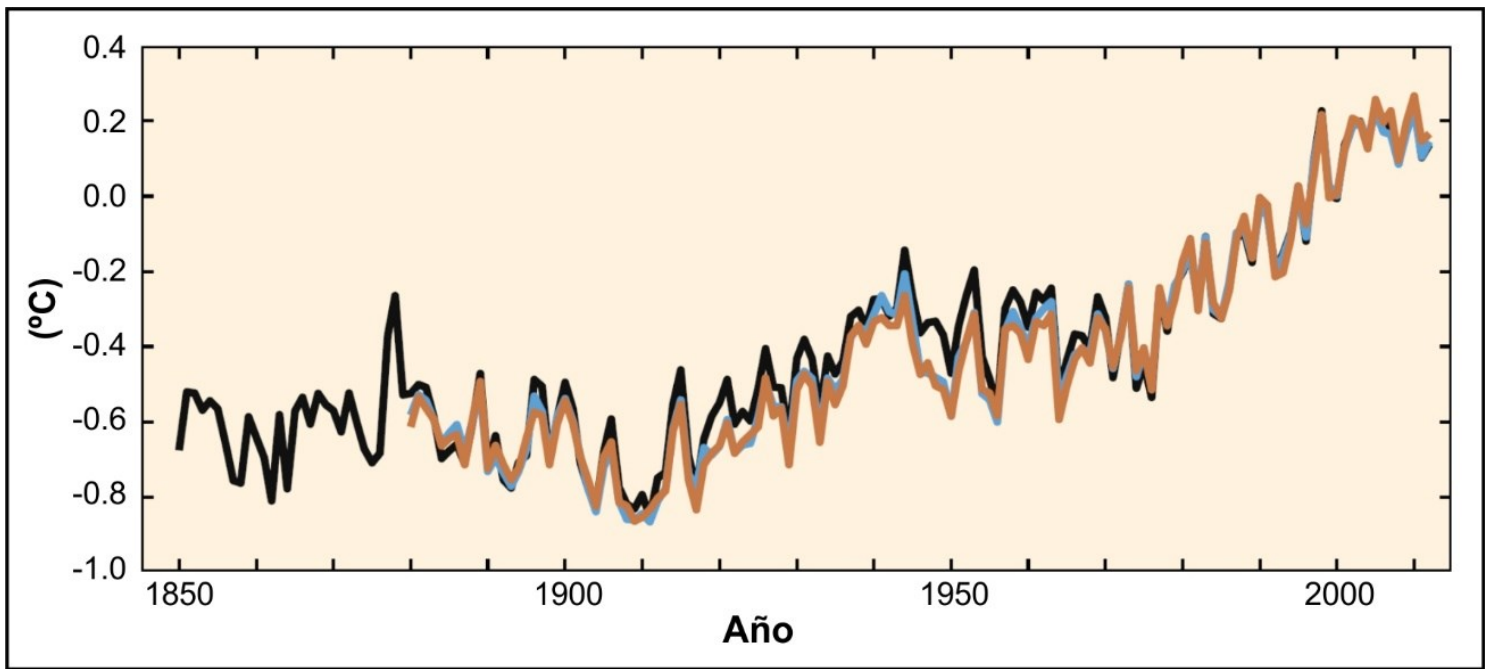

Figura 1. Anomalías del promedio anual y global de temperaturas en superficie, terrestres y oceánicas combinadas respecto del promedio del periodo de 1986 a 2005. Fuente: Panel Intergubernamental de Cambio Climático (2014).

El calentamiento del océano domina sobre el incremento de la energía almacenada en el sistema climático y representa más de $90 \%$ de la energía acumulada entre 1971 y 2010 (nivel de confianza alto), siendo sólo en torno al $1 \%$ la energía almacenada en la atmósfera. A escala global, el calentamiento del océano es mayor cerca de la superficie. Los $75 \mathrm{~m}$ superiores se han calentado $0.11(0.09-0.13){ }^{\circ} \mathrm{C}$ por decenio, durante el periodo comprendido entre 1971 y 2010.

En el periodo comprendido entre 1992 y 2011, los mantos de hielo de Groenlandia y la Antártida han ido perdiendo masa (nivel de confianza alto), y es probable que esa pérdida se haya producido a un 
Tecnología y

Ciencias $\cong$ Agua
2020, Instituto Mexicano de Tecnología del Agua

Open Access bajo la licencia CC BY-NC-SA 4.0

(https://creativecommons.org/licenses/by-nc-sa/4.0/)

ritmo más rápido entre 2002 y 2011. Los glaciares han continuado menguando en casi todo el mundo (nivel de confianza alto).

\section{Causas del cambio climático}

Las emisiones antrópicas acumuladas de gases de efecto invernadero (GEI) desde la era preindustrial han experimentado grandes aumentos en las concentraciones atmosféricas de dióxido de carbono $\left(\mathrm{CO}_{2}\right)$, metano $\left(\mathrm{CH}_{4}\right)$ y óxido nitroso $\left(\mathrm{N}_{2} \mathrm{O}\right)$. Entre 1750 y 2011, las emisiones antrópicas de $\mathrm{CO}_{2}$ a la atmósfera acumuladas fueron de $2040 \pm 310 \mathrm{Gt}$ $\mathrm{CO}_{2}$ (Butze, 2004).

Alrededor de $40 \%$ de esas emisiones ha permanecido en la atmósfera ( $880 \pm 35 \mathrm{Gt} \mathrm{CO}_{2}$ ) y el resto fue removida de la atmósfera y almacenada en la tierra (en plantas y suelos), y en el océano. Los océanos han absorbido alrededor de $30 \%$ del $\mathrm{CO}_{2}$ antrópico emitido, provocando su acidificación. Alrededor de la mitad de estas emisiones acumuladas entre 1750 y 2011 se han producido en los últimos 40 años (nivel de confianza alto).

Las emisiones antrópicas totales de GEI han seguido aumentando entre 1970 y 2010, con mayores incrementos absolutos entre 2000 y 2010, a pesar del creciente número de políticas de mitigación del cambio climático. Las emisiones antrópicas de GEI en 2010 alcanzaron 
la cifra de $49 \pm 4.5 \mathrm{Gt} \mathrm{CO}$-eq/año. Las emisiones de $\mathrm{CO}_{2}$ procedente de la combustión de combustibles fósiles y los procesos industriales contribuyeron en torno al $78 \%$ del aumento total de emisiones de GEI de 1970 a 2010, con una contribución porcentual similar para el aumento experimentado de 2000 a 2010 (nivel de confianza alto). En el mundo, el crecimiento económico y demográfico continuaron siendo los motores más importantes de los aumentos en las emisiones de $\mathrm{CO}_{2}$ derivadas de la quema de combustibles fósiles.

Desde el Cuarto Informe de Evaluación (Panel Intergubernamental del Cambio Climático, 2007) ha aumentado la evidencia de la influencia humana en el sistema climático. Es sumamente probable que más de la mitad del aumento observado en la temperatura media global en superficie de 1951 a 2010 haya sido causado por la combinación del incremento de las concentraciones de gases de efecto invernadero y de otros forzamientos antrópicos.

De acuerdo con las mejores estimaciones, la contribución de la actividad humana al calentamiento es similar al calentamiento observado durante el mencionado periodo. Es probable que los forzamientos antrópicos hayan contribuido de modo considerable a los aumentos de la temperatura en superficie desde mediados del siglo XX en todas las regiones continentales, excepto la Antártida.

Es probable que la influencia antrópica haya afectado al ciclo global del agua desde 1960 y haya contribuido al retroceso de los glaciares desde la década de 1960, así como al aumento del deshielo del manto de hielo de Groenlandia desde 1993. Es muy probable que la 
Tecnología y

Ciencias $\approx$ Agua
2020, Instituto Mexicano de Tecnología del Agua

Open Access bajo la licencia CC BY-NC-SA 4.0

(https://creativecommons.org/licenses/by-nc-sa/4.0/)

influencia antrópica haya contribuido a la pérdida de hielo marino en el Ártico desde 1979 y que haya contribuido de forma significativa a incrementos en el contenido global de calor en la capa superior del océano (0-700 m), así como a la elevación del nivel medio global del mar observado desde la década de 1970 (Panel Intergubernamental de Cambio Climático, 2014).

\section{Temperatura y retroceso glaciar}

Los glaciares pasan por un deshielo global desde hace más de cien años. Michael Zemp, líder del Servicio de Monitoreo Mundial de Glaciares (WGMS), sostiene que los glaciares observados pierden hoy entre medio metro y un metro de espesor de hielo cada año, caso que representa dos o tres veces más que el promedio del siglo pasado (Gubin, 2015). El manto de nieve en primavera en el hemisferio norte sigue reduciéndose. Existe un nivel de confianza alto en cuanto a que las temperaturas del permafrost han aumentado en la mayoría de las regiones desde principios de la década de 1980, en respuesta a la subida de la temperatura en superficie y a la alteración del manto de nieve.

En el Ártico, es muy probable que la superficie media anual del hielo marino haya disminuido de 1979 a 2012, en un rango de 3.5 a $4.1 \%$ por decenio. La extensión del hielo marino en la zona ha 
Tecnología y

Ciencias $\approx$ Agua
2020, Instituto Mexicano de Tecnología del Agua

Open Access bajo la licencia CC BY-NC-SA 4.0

(https://creativecommons.org/licenses/by-nc-sa/4.0/)

disminuido en cada estación y en cada decenio sucesivo desde 1979, y es en verano cuando se ha registrado el mayor ritmo de disminución en la extensión media decenal.

En la Antártida, es muy probable que la extensión media anual del hielo marino haya aumentado en un rango de entre 1.2 y $1.8 \%$ por decenio entre 1979 y 2012; sin embargo, existe un nivel de confianza alto respecto a que existen marcadas diferencias regionales en este continente, con un aumento de la extensión en algunas regiones y una disminución en otras. De 1901 a 2010, el nivel medio global del mar se elevó $0.19 \mathrm{~m}$ (de 0.17 a $0.21 \mathrm{~m}$ ). Desde mediados del siglo XIX, el ritmo de la elevación del nivel del mar ha sido superior a la media de los dos milenios anteriores (Panel Intergubernamental de Cambio Climático, 2014).

El retroceso de la masa glaciar en las montañas chinas, por ejemplo, está identificado como muy vulnerable al cambio climático, sobre todo en el oeste de China. De 1961 a 2007, un 92\% de las zonas de glaciares tenía una vulnerabilidad leve; hoy, $41.2 \%$ de tales áreas está en los niveles fuerte y muy fuerte de vulnerabilidad.

En general, la vulnerabilidad de los glaciares muestra una tendencia decreciente entre 2030 y el 2050; pero los glaciares en Altai, Tianshan, Kunlun, las zonas central y occidental de la montaña Qilian, zona central y zona oriental del Himalaya, y el sureste del Tíbet seguirán siendo clasificadas en los niveles fuerte y muy fuertes de vulnerabilidad.

Los glaciares de China eran relativamente muy vulnerables en el periodo 1961-2007, y se considera que la topografía y la alta 
Tecnología y

Ciencias $\approx$ Agua
2020, Instituto Mexicano de Tecnología del Agua

Open Access bajo la licencia CC BY-NC-SA 4.0

(https://creativecommons.org/licenses/by-nc-sa/4.0/)

sensibilidad de los glaciares al cambio climático son las razones de tal situación. Además de los factores topográficos, se considera que las variaciones en las precipitaciones podrían convertirse en un factor crucial que afecta la vulnerabilidad de los glaciares en los años 2030 y 2050 (Jian-Ping, Yong-Jian, Shi-Yin, \& Chun-Ping, 2015).

En la morfología de los ecosistemas de las altas montañas están registrándose los impactos negativos a los glaciares tropicales, denominado retroceso glaciar, y aparición de nuevas lagunas en las concavidades dejadas por la masa de hielo. Los cambios de las variaciones de las coberturas de glaciares y lagunas se evidencian en las 19 cordilleras nevadas del Perú, divididas en tres sectores: Norte, Centro y Sur, mostrando un total de 2679 glaciares, con una superficie de $1298.59 \mathrm{~km}^{2}$.

Las cordilleras de mayor extensión son las siguientes: la Cordillera Blanca (Andes del Norte), Vilcanota y Vilcabamba (Andes del Centro), con 755, 374 y 355 glaciares y superficies de $527.62 ; 279.40$ y 129.15 $\mathrm{km}^{2}$, respectivamente. En general, la altitud mínima de los glaciares inventariados se encuentra sobre los $4000 \mathrm{msnm}$. Las cordilleras nevadas del Perú en los últimos 40 años han registrado una pérdida en superficie de $42.64 \%$, con respecto a los resultados obtenidos en el inventario del año 1970. Los glaciares pequeños son los más susceptibles a los cambios de superficie; en el último inventario realizado se identificó un total de 2341 glaciares $(87.38 \%)$ con superficie $\leq 1 \mathrm{~km}^{2}$ y 338 glaciares $(12.62 \%)$ con superficie $>1 \mathrm{~km}^{2}$. Los resultados obtenidos del inventario de lagunas de origen glaciar suman un total de 8355 lagunas, que se ubican en el ámbito de las 19 Tecnología y ciencias del agua, ISSN 2007-2422, 11(2), 229-261. DOI: 10.24850/j-tyca-2020-02-06 
Tecnología y

Ciencias $\approx$ Agua
2020, Instituto Mexicano de Tecnología del Agua

Open Access bajo la licencia CC BY-NC-SA 4.0

(https://creativecommons.org/licenses/by-nc-sa/4.0/)

cordilleras nevadas del país, y cubren una superficie de $916.64 \mathrm{~km}^{2}$ (Autoridad Nacional del Agua, 2014).

Entre 2003 y 2011, las mediciones de balance de masa realizadas en los glaciares Yanamarey y Artesonraju en la Cordillera Blanca, en Huaraz, mostraron en el caso del Yanamarey pérdidas de área en el frente y en los bordes laterales hasta la parte alta del glaciar. A pesar de la desaceleración del aumento de la temperatura y un aumento en la precipitación, la retirada de los glaciares ha continuado a un ritmo elevado en los últimos treinta años.

La precipitación y la temperatura pueden afectar el proceso de acumulación. El aumento de la precipitación observada durante las estaciones húmedas conduciría a un aumento de la precipitación sólida en el área de acumulación $y$, por tanto, un balance de masas más positivo anual en caso de precipitación que caiga en forma de nieve (Vuille, Kaser, \& Juen, 2008); por ejemplo, se encontró que en escalas de tiempo interanuales, la variabilidad de la precipitación parece ser el principal motor de las fluctuaciones de la masa glaciar en la Cordillera Blanca. Por otro lado, el aumento de temperaturas de aire durante los episodios de precipitaciones conduce también a un aumento de la línea de nieve. Sin embargo, el incremento de la temperatura del aire en los últimos treinta años es particularmente dominante en la temporada de junio a noviembre, relativamente seca, en la que las precipitaciones son más bien escasas.

Las precipitaciones en la Cordillera Blanca han aumentado de modo significativo entre 1980 y 2012, lo que llevaría a un balance de 
Tecnología y

Ciencias $\approx$ Agua
2020, Instituto Mexicano de Tecnología del Agua

Open Access bajo la licencia CC BY-NC-SA 4.0

(https://creativecommons.org/licenses/by-nc-sa/4.0/)

masa más positivo si la precipitación cae en forma de nieve, condición que evidencia que los glaciares han continuado retrocediendo desde la década de 1980. La disminución de la superficie glaciar es particularmente alta para los glaciares de baja altitud y glaciares aislados, como el Yanamarey y Pastoruri.

Hay estudios que informan de los diferentes escenarios de retiro para los pequeños glaciares con elevaciones máximas debajo de 5400 msnm y los grandes glaciares con la máxima elevación por encima de tal altitud. Los cambios de temperatura y precipitación desde el decenio de 1980 probablemente no explican por completo el fuerte retroceso de los glaciares durante los últimos treinta años. Por ello es posible que el reciente retroceso de los glaciares aún puede ocurrir en respuesta a la fuerte subida de temperatura de más de $0.3^{\circ} \mathrm{C}$ por década antes de 1980, sobre todo en el decenio de 1970.

En general, los glaciares con bajo espesor del hielo en la línea de equilibrio y grande ablación anual en la lengua del glaciar tienen tiempos de respuesta menores a perturbaciones climáticas que los grandes glaciares. Existen mediciones y estimaciones de espesor de hielo y tasas de ablación anuales para algunos glaciares en la Cordillera Blanca (p. ej., Artesonraju) y permiten estimar un tiempo de respuesta del orden de 10 a 40 años.

El fuerte retroceso de los glaciares observado durante las últimas tres décadas puede incluir una señal del aumento de la temperatura antes de la década de 1980, en función del glaciar. La subida de 
Tecnología y

Ciencias $₫$ Agua
2020, Instituto Mexicano de Tecnología del Agua

Open Access bajo la licencia CC BY-NC-SA 4.0

(https://creativecommons.org/licenses/by-nc-sa/4.0/)

temperatura moderada durante los últimos treinta años puede haber inducido un forzamiento adicional.

Sin embargo, la interpretación de las respuestas de los glaciares de forzamiento climático es un reto, pues algunas fluctuaciones climáticas ocurren en escalas de tiempo más corto que los tiempos de reacción y, en consecuencia, la respuesta observada de un glaciar puede ser una reacción a un gran número de causas superpuestas (Schauwecker et al., 2014).

\section{EI retroceso glaciar en la cordillera Huaytapallana}

En el caso de la cordillera Huaytapallana, Arroyo (2013), y Arroyo, Gurmendi y Machuca (2015) comunican que: "se reporta en los estudios de Zubieta y Lagos (15), que hubo una pérdida neta superior al 50\% con respecto a 1956. Las anomalías negativas producto del fenómeno de El Niño aceleran la disminución de los glaciares y que tiene como consecuencia el enfriamiento del sistema que ocasiona anomalías positivas y genera un aumento de la masa glaciar temporal"; asimismo, Zubieta y Lagos (2010) mencionan que "entre 1976 y 2006, la superficie glaciar de la Cordillera Huaytapallana se redujo de 35.6 a $14.5 \mathrm{~km}^{2}$, esto representa una pérdida del 59.4\%, a su vez la dinámica espacial del retiro de glaciares —ocurrido en los circos del Huaytapallana- también 
Tecnología y

Ciencias
2020, Instituto Mexicano de Tecnología del Agua

Open Access bajo la licencia CC BY-NC-SA 4.0

(https://creativecommons.org/licenses/by-nc-sa/4.0/)

está sujeta a la morfología y dirección de sus masas glaciares, por ello, la marcada diferencia en la distribución del retiro en los glaciares de circo" (IGP, 2012, p. 85).

La tendencia de decrecimiento de la superficie glaciar también es mencionado por López-Moreno et al. (2014) al puntualizar que el retroceso glaciar sería de $55 \%$ en los últimos 28 años, y que sus impactos podrían ser significativos respecto a los recursos hídricos y la ecología del área.

La Autoridad Nacional del Agua (2014, p. 17) reporta que la Cordillera Huaytapallana —cuya máxima elevación es el nevado del mismo nombre- ha perdido $58.4 \%$ de su masa glaciar, según el Inventario de Glaciares y Lagunas presentado en el mismo año; a la vez, las 19 cordilleras nevadas del Perú, identificadas en el mencionado informe, han perdido, en conjunto, $42.6 \%$ de su superficie glaciar; el caso de la laguna Lazuntay sería un ejemplo de esta tendencia en la Cordillera Huaytapallana. Al existir carencia de otros estudios relevantes con mediciones anuales, los medios de comunicación también han difundido información de las fuentes antes mencionadas, tal es el caso de La República (2014), que menciona datos del informe de la Autoridad Nacional del Agua del Perú.

\section{Planteamiento del estudio}


Tecnología y

Ciencias
2020, Instituto Mexicano de Tecnología del Agua

Open Access bajo la licencia CC BY-NC-SA 4.0

(https://creativecommons.org/licenses/by-nc-sa/4.0/)

El estudio se ha desarrollado basado en la pregunta de investigación ¿cómo influye el cambio de temperatura ambiental como manifestación del cambio climático en el retroceso glaciar de la Cordillera Huaytapallana, Perú? Se han planteado como objetivos describir el efecto de la temperatura ambiental en los últimos treinta años y su relación con el retroceso de la superficie glaciar de la Cordillera Huaytapallana.

Este estudio se justifica en atención a los compromisos asumidos por los países firmantes del Protocolo de Kioto, establecido en el Convenio Marco sobre el Cambio Climático, y que entre otros asuntos plantea la necesidad de cooperar en investigaciones para reducir las incertidumbres relacionadas con el cambio climático, así como facilitar el conocimiento y el acceso público a la información sobre el cambio climático (ONU, 1997). En este contexto, nuestro país ratificó el Protocolo de Kioto en 2002, y remitió de forma oportuna su ofrecimiento para la mitigación global a través de su "Contribución Prevista y Determinada a Nivel Nacional" (INDC, por sus siglas en inglés) en septiembre de 2015, y participó en los esfuerzos para alcanzar el Acuerdo de París en diciembre de 2015 (Ministerio del Ambiente del Perú, 2016).

\section{Materiales y métodos}




\section{Descripción del área de estudio}

El departamento de Junín tiene una población de 1331253 personas. La provincia de Huancayo es la capital, con una población de 499432 personas, tiene 28 distritos, y el distrito de Huancayo tenía una población estimada de 116930 habitantes al 2013 (INEI, 2015; Banco Central de Reserva del Perú, 2011). Asimismo, la superficie de la provincia es de $3558.1 \mathrm{~km}^{2}$; está ubicada a $11^{\circ}$ latitud sur y $77^{\circ}$ longitud oeste, a una altura de 3194 msnm (Wikipedia, 2016).

Las mediciones de la superficie glaciar fueron realizadas en la Cordillera Huaytapallana, ubicada en la provincia de Huancayo, entre las coordenadas $11^{\circ} 35^{\prime}-11^{\circ} 58^{\prime}$ de latitud sur y $74^{\circ} 48^{\prime}-75^{\circ} 17^{\prime}$ de longitud oeste; presentaba 105 glaciares, y una superficie de $14.3 \mathrm{~km}^{2}$ al año 2011 (IGP, 2012).

\section{Aspectos metodológicos}


Tecnología y

Ciencias $\approx$ Agua
2020, Instituto Mexicano de Tecnología del Agua

Open Access bajo la licencia CC BY-NC-SA 4.0

(https://creativecommons.org/licenses/by-nc-sa/4.0/)

El tipo de investigación es básica, porque genera conocimiento; de enfoque cuantitativo, al haber planteado hipótesis, realizado mediciones y analizado de manera cuantitativa las variables de estudio (HernándezSampieri, Fernández-Collado, \& Baptista-Lucio, 2014). El método general de investigación fue analítico-sintético; de alcance descriptivocorrelacional, con un diseño no experimental, porque no se manipuló variable alguna, y transversal por haber recolectado datos en un solo momento (Hernández-Sampieri et al., 2014).

Los datos de temperatura mínima -como manifestación del cambio climático- y superficie glaciar se obtuvieron a partir de informes oficiales del Servicio Nacional de Meteorología e Hidrología (Senamhi, 2016) y del Observatorio de Huancayo, perteneciente al Instituto Geofísico del Perú (IGP, 2016). Los datos de temperatura mínima están expresadas en ${ }^{\circ} \mathrm{C}$ y los datos de superficie glaciar en $\mathrm{km}^{2}$. Los datos de temperatura pertenecen al Observatorio de Huancayo, ubicado en latitud $12^{\circ} 02^{\prime} \mathrm{S}$, longitud $75^{\circ} 19^{\prime} \mathrm{W}$, altitud $3313 \mathrm{msnm}$; en el distrito de Huachac, provincia de Chupaca, departamento de Junín, Perú. Los datos de superficie glaciar se tomaron de estudios de la Cordillera Huaytapallana, por ser la más cercana de los glaciares a la zona de estudio.

Para la estimación de los datos faltantes de las variables de estudio en determinados años se exploró la dispersión de los datos de las variables temperatura mínima y superficie glaciar, consideradas como variables dependientes con respecto al año como variable independiente; luego se seleccionaron modelos de regresión sobre la 
Tecnología y

Ciencias $\approx$ Agua
2020, Instituto Mexicano de Tecnología del Agua

Open Access bajo la licencia CC BY-NC-SA 4.0

(https://creativecommons.org/licenses/by-nc-sa/4.0/)

base de la significancia de los parámetros y el coeficiente de determinación $\left(R^{2}\right)$; el modelo lineal se escogió para las variables temperatura mínima y superficie glaciar.

El modelo matemático aplicado se formula como: $Y=\beta_{0}+\beta_{1} X+$ $\varepsilon$, donde $Y$ es la variable dependiente (superficie glaciar); $X$, la variable independiente (temperatura mínima); $\beta_{0}$, el intercepto; $\beta_{1}$, la pendiente de la regresión, y $\varepsilon$ es el error o residuo.

Los datos no disponibles entre $1986-2016$ se interpolaron sobre la base de modelos de regresión lineal. Para determinar la normalidad de las variables dependientes se utilizó la prueba de Shapiro-Wilk, luego la relación de las variables independientes y dependientes se realizó con la prueba $r$ de Pearson. Para la validación de los modelos de regresión lineal se utilizó el análisis de varianza y prueba de Durbin Watson.

\section{Resultados}

El cambio climático se evidencia en la ciudad de Huancayo con base en el incremento del indicador de temperatura mínima del periodo 19862016 (30 años). Los valores anuales son la media de los valores mínimos mensuales registrados por el Observatorio de Huancayo del IGP; asimismo, el comportamiento de los glaciares de la Cordillera 
Tecnología y

Ciencias $₫$ Agua
2020, Instituto Mexicano de Tecnología del Agua

Open Access bajo la licencia CC BY-NC-SA 4.0

(https://creativecommons.org/licenses/by-nc-sa/4.0/)

Huaytapallana, que en el mismo periodo viene mostrando un retroceso de la masa glaciar.

Entre 1986 y 2016 se evidencia un aumento para las temperaturas mínima y media anual en la zona de estudio; este incremento es más alto para la temperatura mínima, mostrando valores de 3.435 a 5.227 ${ }^{\circ} \mathrm{C}$; se estima al 2016 un valor de $4.757^{\circ} \mathrm{C}$ (Figura 2); de igual forma, la superficie de la masa glaciar de la Cordillera Huaytapallana muestra en este mismo periodo una tendencia de retroceso, estimado al 2016 en $11.86 \mathrm{~km}^{2}$ de extensión (Figura 2)

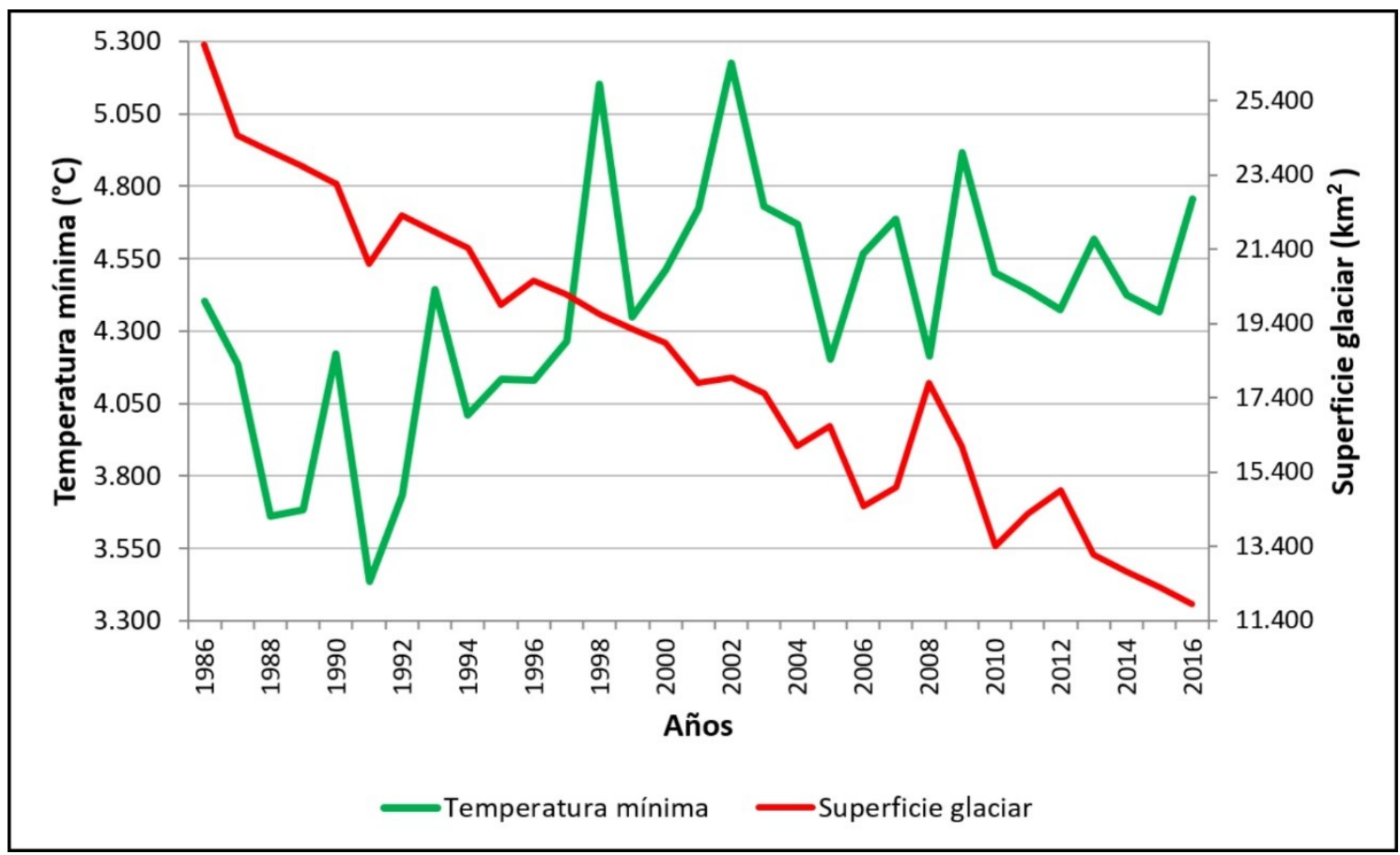

Figura 2. Datos observados y estimados de temperatura mínima y superficie glaciar de la Cordillera de Huaytapallana de 1986 a 2016, Huancayo, Perú. Datos del Senamhi (2016), IGP (2016). Fuente: 
Tecnología y

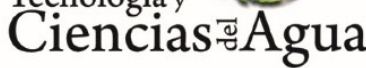

2020, Instituto Mexicano de Tecnología del Agua

Open Access bajo la licencia CC BY-NC-SA 4.0

(https://creativecommons.org/licenses/by-nc-sa/4.0/)

elaboración propia.

Para la comprobación de las hipótesis estadísticas se plantearon las siguientes:

$H_{0}$ : no existe relación entre temperatura y superficie glaciar como dimensiones del cambio climático en Huancayo al $2016\left(H_{0}: \rho=\right.$ $0)$.

$H_{1}$ : hay relación significativa entre temperatura y superficie glaciar como dimensiones del cambio climático en Huancayo al $2016\left(H_{1}\right.$ : $\rho \neq 0)$.

La Tabla 1 muestra los resultados de la aplicación de la prueba estadística Shapiro-Wilk a la variable superficie glaciar, cuya significancia indica que las muestras tienen una distribución normal (0.641 es mayor a $a=0.05)$.

Tabla 1. Prueba de normalidad de superficie glaciar.

\begin{tabular}{|l|c|c|c|}
\hline & \multicolumn{3}{|c|}{ Shapiro-Wilk } \\
\hline & Estadístico & gl & Sig. \\
\hline Superficie glaciar & 0.974 & 31 & 0.641 \\
\hline
\end{tabular}

Fuente: elaboración propia. 
Tecnología y

Ciencias $\approx$ Agua
2020, Instituto Mexicano de Tecnología del Agua

Open Access bajo la licencia CC BY-NC-SA 4.0

(https://creativecommons.org/licenses/by-nc-sa/4.0/)

En la Figura 3 se observa que la muestra de puntuaciones se distribuye normalmente, pues los puntos del diagrama $Q-Q$ normal se ajustan a la recta diagonal.

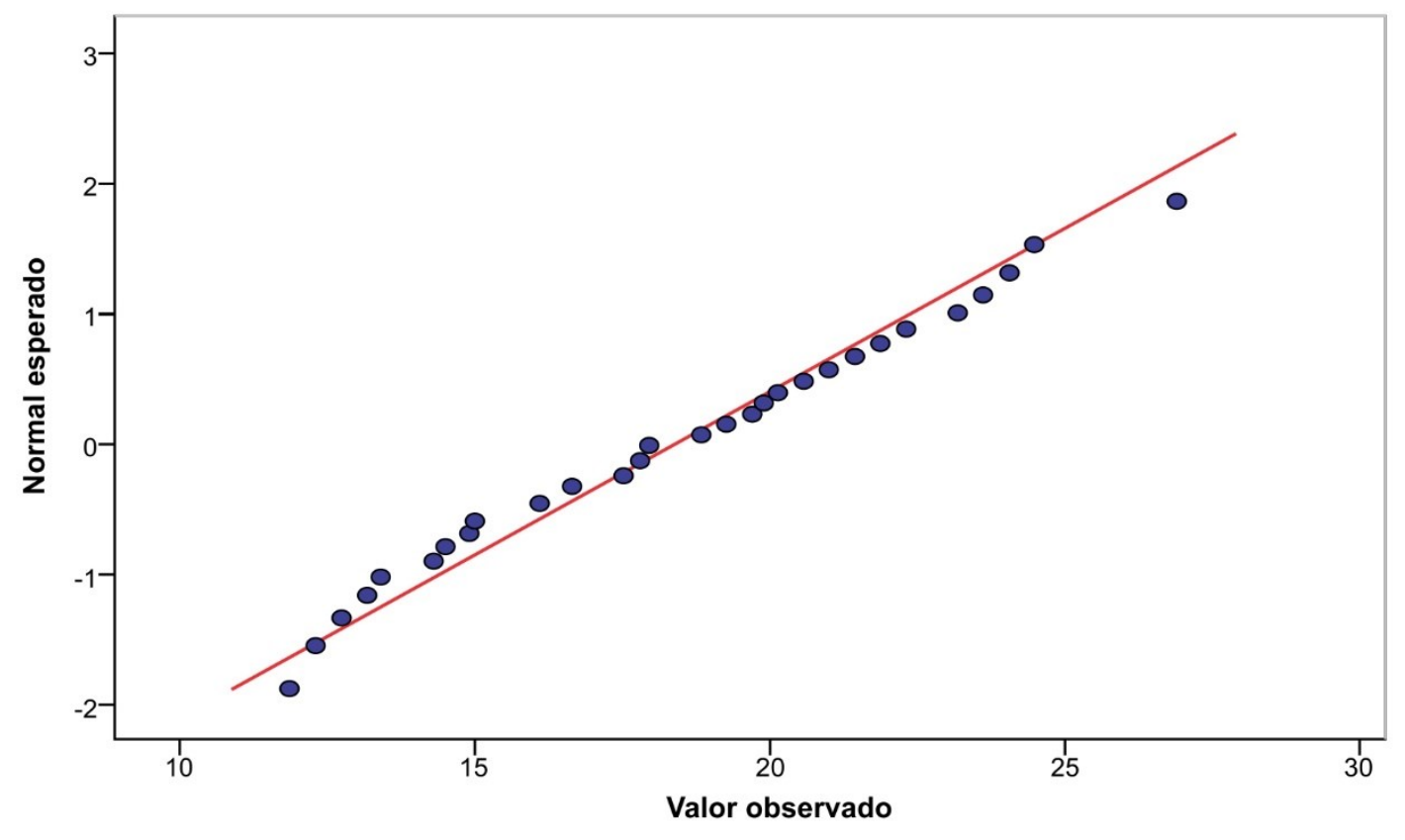

Figura 3. Gráfico $Q-Q$ normal de superficie glaciar. Fuente: elaboración propia.

En la Tabla 2 y Figura 4 se muestra que la correlación entre la temperatura mínima y superficie glaciar es negativa y moderada ($\left.0.493^{* *}\right)$ altamente significativa (0.002 es menor a 0.05$)$.

Tabla 2. Correlación de temperatura y superficie glaciar. 
Tecnología y

Ciencias $₫$ Agua
2020, Instituto Mexicano de Tecnología del Agua

Open Access bajo la licencia CC BY-NC-SA 4.0

(https://creativecommons.org/licenses/by-nc-sa/4.0/)

\begin{tabular}{|l|l|r|}
\hline \multirow{2}{*}{$\begin{array}{l}\text { Temperatura } \\
\text { mínima }\end{array}$} & Correlación de Pearson & Superficie glaciar \\
\cline { 2 - 3 } & Sig. (unilateral) & $-0.493^{* *}$ \\
\hline & $\mathrm{N}$ & 0.002 \\
\hline ** La correlación es significativa en el nivel 0.01 (unilateral) \\
\hline
\end{tabular}

Fuente: elaboración propia.

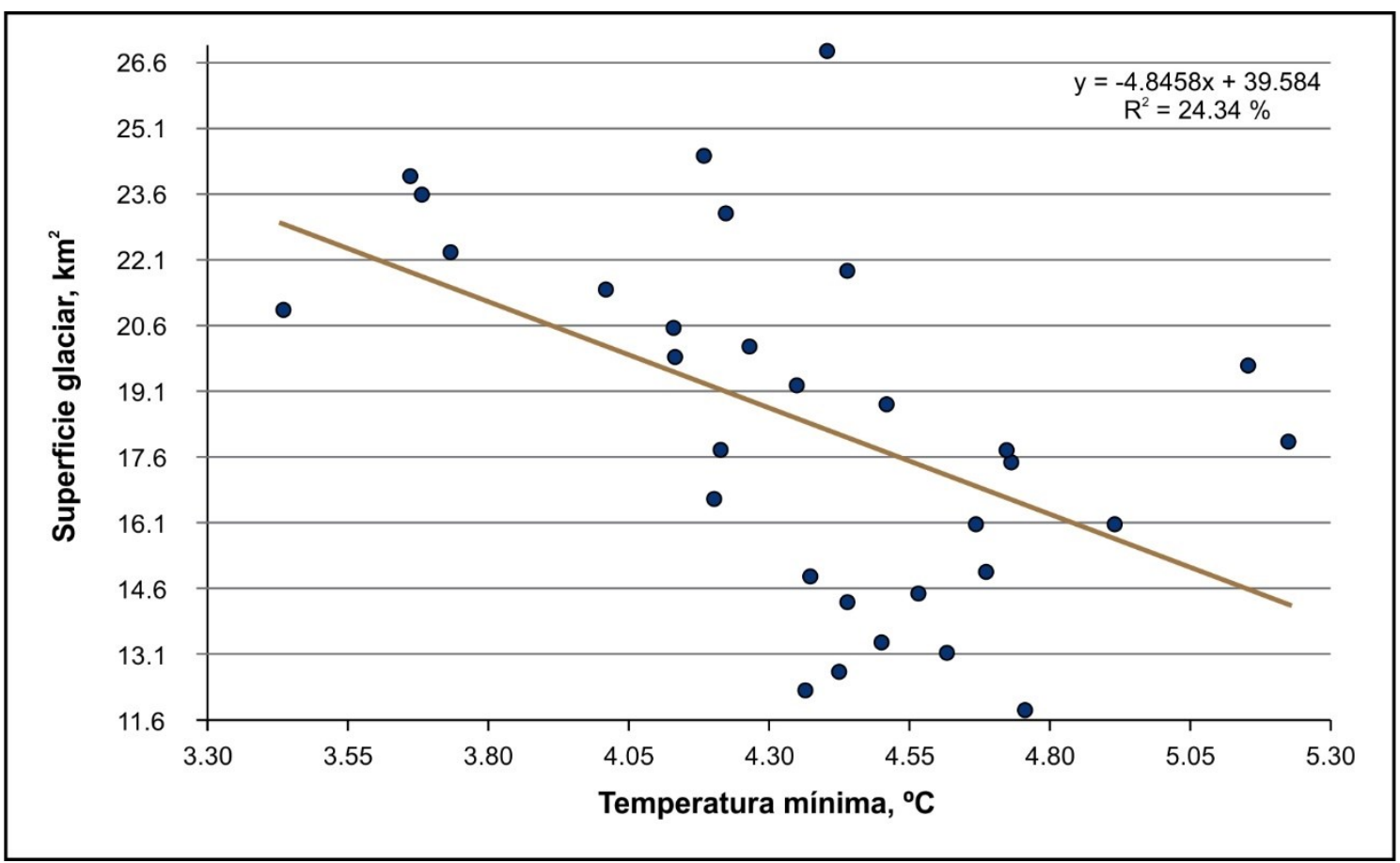

Figura 4. Relación temperatura mínima y superficie glaciar. Fuente: elaboración propia.

En la Tabla 3 se muestra el resumen del modelo de regresión lineal simple aplicado, donde la variable incluida (temperatura mínima) 
Tecnología y

Ciencias $\approx$ Agua
2020, Instituto Mexicano de Tecnología del Agua

Open Access bajo la licencia CC BY-NC-SA 4.0

(https://creativecommons.org/licenses/by-nc-sa/4.0/)

en el análisis explica un $24.3 \%$ de la varianza de la variable dependiente (superficie glaciar) $\left(R^{2}=24.3 \%\right)$; el error típico de los residuos es 3.546; el estadístico Durbin-Watson (0.473) indica que existe autocorrelación positiva entre los residuos.

Tabla 3. Modelo de regresión lineal simple entre temperatura mínima y superficie glaciar.

\begin{tabular}{|c|c|c|c|c|}
\hline Modelo & $\boldsymbol{R}$ & $\begin{array}{c}\boldsymbol{R} \\
\text { cuadrado }\end{array}$ & $\begin{array}{c}\text { Error estándar } \\
\text { de la estimación }\end{array}$ & $\begin{array}{c}\text { Durbin- } \\
\text { Watson }\end{array}$ \\
\hline 1 & $0.493^{\mathrm{a}}$ & $24.3 \%$ & 3.54854 & 0.473 \\
\hline
\end{tabular}

Fuente: elaboración propia.

En la Tabla 4 se aprecia el resultado del análisis de varianza de la regresión simple aplicada con la prueba $\mathrm{F}$ a nivel $(a=0.05)$, donde la significancia del estadístico $F(0.005)$ indica que existe relación lineal altamente significativa entre las variables.

Tabla 4. Análisis de varianza para la regresión lineal simple entre temperatura mínima y superficie glaciar.

\begin{tabular}{|l|l|c|c|c|c|c|}
\hline Modelo & $\begin{array}{c}\text { Suma de } \\
\text { cuadrados }\end{array}$ & GI & $\begin{array}{c}\text { Media } \\
\text { cuadrática }\end{array}$ & F & Sig. \\
\hline
\end{tabular}


Tecnología y

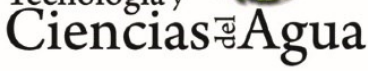

2020, Instituto Mexicano de Tecnología del Agua

Open Access bajo la licencia CC BY-NC-SA 4.0

(https://creativecommons.org/licenses/by-nc-sa/4.0/)

\begin{tabular}{|c|c|c|c|c|c|c|}
\hline \multirow[t]{3}{*}{1} & Regresión & 117.464 & 1 & 117.464 & 9.328 & $0.005^{b}$ \\
\hline & Residuo & 365.171 & 29 & 12.592 & & \\
\hline & Total & 482.635 & 30 & & & \\
\hline $\bar{a}=v_{c}$ & dependie & superficie $c$ & iar. & & & \\
\hline
\end{tabular}

Fuente: elaboración propia.

La Tabla 5 muestra los coeficientes del modelo de regresión lineal simple ajustado, descrito como la superficie glaciar $(Y)=39.587$ $4.846 *$ temperatura.

Tabla 5. Coeficientes y significación estadística de la variable superficie glaciar.

\begin{tabular}{|c|c|c|c|c|c|}
\hline & \multicolumn{2}{|c|}{$\begin{array}{c}\text { Coeficientes no } \\
\text { estandarizados }\end{array}$} & $\begin{array}{c}\text { Coeficientes } \\
\text { estandarizados }\end{array}$ & Error \\
\hline Modelo & B & $\begin{array}{c}\text { Beta } \\
\text { estándar }\end{array}$ & & Sig. \\
\hline 1 (Constante) & 39.587 & 6.977 & & 5.674 & 0.000 \\
\hline Temperatura & -4.846 & 1.587 & -0.493 & -3.054 & 0.002 \\
\hline a = variable dependiente: superficie glaciar. &
\end{tabular}

Fuente: elaboración propia. 
Tecnología y

Ciencias $₫$ Agua
2020, Instituto Mexicano de Tecnología del Agua

Open Access bajo la licencia CC BY-NC-SA 4.0

(https://creativecommons.org/licenses/by-nc-sa/4.0/)

Los coeficientes estimados del modelo $\left(b_{0}=39.587\right.$ y $b_{1}=$ 4.846) son altamente significativos ( $\mathrm{Sig}<0.01$ ), con los cuales se afirma que es estadísticamente válido. El coeficiente 39.587 significa que si la temperatura aumenta en $1^{\circ} \mathrm{C}$, la superficie glaciar disminuye en $4.846 \mathrm{~km}^{2}$.

En conclusión, al 99\% de confianza estadística, se concluye que existe relación lineal altamente significativa entre temperatura y superficie glaciar al 2016; asimismo, el coeficiente de determinación $\left(R^{2}\right)$ es bajo (de 0.2 a 0.4 ).

\section{Discusión}

La temperatura en Huancayo en los últimos años muestra una tendencia de incremento (Baltazar-Castañeda, 2014). La temperatura mínima estimada en Huancayo al $2016\left(4.757^{\circ} \mathrm{C}\right)$ evidencia mejor el cambio del clima, factor que contribuye con el retroceso de la masa glaciar de la Cordillera Huaytapallana; de acuerdo con las mediciones efectuadas entre 1986-2016 y estimaciones basadas en modelos de regresión lineal, se estima la superficie glaciar en $11.86 \mathrm{~km}^{2}$ al 2016; la relación entre estas variables es lineal, inversa y moderada; es evidente que el incremento de la temperatura incide en el retroceso de los glaciares de 
Tecnología y

Ciencias
2020, Instituto Mexicano de Tecnología del Agua

Open Access bajo la licencia CC BY-NC-SA 4.0

(https://creativecommons.org/licenses/by-nc-sa/4.0/)

la Cordillera Huaytapallana, coincidiendo este fenómeno con el caso de la Cordillera Blanca en Ancash, Perú, reportado por la Autoridad Nacional del Agua (2014).

Al respecto, Gonzáles (2013) afirma que las altas concentraciones de $\mathrm{CO}_{2}$ y otros GEI en la atmósfera generan el incremento de la temperatura superficial promedio en el planeta; asimismo, éste sería uno de los principales factores del retroceso glaciar.

El Panel Intergubernamental de Cambio Climático (2014) respecto a los glaciares en los polos de la Tierra, caso del Ártico, reporta como muy probable que la superficie media anual del hielo marino haya disminuido durante el periodo $1979-2012$, en un rango de 3.5 a $4.1 \%$ por decenio. La extensión del hielo marino en la zona ha disminuido en cada estación y en cada decenio sucesivo desde 1979, y es en verano cuando se ha registrado el mayor ritmo de disminución en la extensión media decenal.

También en cuanto a los resultados, coinciden con los reportados por Schauwecker et al. (2014), que manifiestan que un fuerte retroceso de los glaciares, observado durante las últimas tres décadas, puede incluir una señal del aumento de la temperatura antes del decenio de 1980, en función del glaciar. La subida de temperatura moderada durante los últimos treinta años puede haber inducido un forzamiento adicional.

A futuro -en términos de sostenibilidad de los recursos naturales-, los recursos hídricos serán insuficientes ante la demanda para el consumo humano y diversas actividades socioeconómicas de la 
Tecnología y

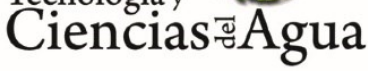

2020, Instituto Mexicano de Tecnología del Agua

Open Access bajo la licencia CC BY-NC-SA 4.0

(https://creativecommons.org/licenses/by-nc-sa/4.0/)

ciudad de Huancayo.

\section{Conclusiones}

La temperatura mínima estimada al 2016 en $4.757^{\circ} \mathrm{C}$ está incidiendo en la reducción de la superficie glaciar de la Cordillera Huaytapallana, que se estima en $11.86 \mathrm{~km}^{2}$ para el mismo año. Existe una relación inversa, moderada y significativa entre el incremento de la temperatura y el retroceso de la masa glaciar de la Cordillera Huaytapallana.

\section{Agradecimientos}

Al Servicio Nacional de Meteorología e Hidrología del Perú (Senamhi) e Instituto Geofísico del Perú (IGP) por el acceso y uso de datos meteorológicos de la zona de estudio; asimismo, a Romina Bulege, por la traducción al inglés.

\section{Referencias}

Arroyo, J. (13 de abril, 2013). Impactos de las actividades antrópicas en el nevado Huaytapallana. Apuntes de Ciencia \& Sociedad, 3-14. Recuperado de 
Tecnología y

Ciencias
2020, Instituto Mexicano de Tecnología del Agua

Open Access bajo la licencia CC BY-NC-SA 4.0

(https://creativecommons.org/licenses/by-nc-sa/4.0/)

http://journals.continental.edu.pe/index.php/apuntes/article/view/ $41 / 40$

Arroyo, J., Gurmendi, P., \& Machuca, E. (2015). Efectos de las anomalías climáticas en la cobertura de nieve de los glaciares centrales del Perú. Apuntes de Ciencia \& Sociedad, 146-156. Recuperado de http://journals.continental.edu.pe/index.php/apuntes/article/view/ $310 / 325$

Autoridad Nacional del Agua. (2014). Inventario de glaciares del Perú (2da. actualización). Huaraz, Perú: Autoridad Nacional del Agua. Recuperado de http://www.ana.gob.pe/media/981508/glaciares.pdf

Baltazar-Castañeda, H. (2014). Factores climáticos que influyen en la diversidad de insectos en Spartium junceum L. (Fabales:

Fabaceae) en el Valle del Mantaro. Huancayo, Perú: Universidad Nacional del Centro del Perú.

Banco Central de Reserva del Perú. (2011). Portal web del BCRP.

Recuperado de

http://www.bcrp.gob.pe/docs/Sucursales/Huancayo/Junin-

Caracterizacion.pdf

Butze, W. (2004). El cambio climático: un problema de energía. El

Cotidiano, 19(123), 66-79. Recuperado de http://www.redalyc.org/pdf/325/32512307.pdf

Gonzáles, D. (2013). Energía y cambio climático. Revista Derecho Ambiental y Ecología, 10(55), 61-63. 
Tecnología y

Ciencias
2020, Instituto Mexicano de Tecnología del Agua

Open Access bajo la licencia CC BY-NC-SA 4.0

(https://creativecommons.org/licenses/by-nc-sa/4.0/)

Gubin, A. (4 de abril, 2015). Glaciares de todo el mundo desaparecen y a velocidad sin precedentes. Recuperado de http://www.lagranepoca.com/cienciay-tecnologia/noticias/14013glaciares -de-todo-el-mundo-desapareceny-a-velocidad-sinprecedentes.html

Hernández-Sampieri, R., Fernández-Collado, C., \& Baptista-Lucio, P. (2014). Metodología de la investigación. México, DF, México: McGraw Hill.

IGP, Instituto Geofísico del Perú. (2012). Eventos meteorológicos extremos (sequías, heladas y lluvias intensas) en el Valle del Mantaro. Lima, Perú: Instituto Geofísico del Perú. Recuperado de http://repositorio.igp.gob.pe/handle/IGP/740

IGP. (5 de 11 de 2016). Instituto Geofísico del Perú. Obtenido de Biblioteca IGP: http://biblioteca.igp.gob.pe/cgi-bin/koha/opacsearch.pl?q=Metereolog\%C3\%ADa

INEI, Instituto Nacional de Estadística e Informática. (21 de noviembre de 2015). INEI. Recuperado de http://www.inei.gob.pe/estadisticas/indice-tematico/poblacion-yvivienda/

Jian-Ping, Y., Yong-Jian, D., Shi-Yin, L., \& Chun-Ping, T. (2015). Vulnerability of mountain glaciers in China to climate change. Advances in Climate Change Research, 6(1). Recuperado de https://doi.org/10.1016/j.accre.2015.11.003

La República. (7 de diciembre, 2014). La caída de un gigante: el nevado Huaytapallana. Recuperado de 
Tecnología y

Ciencias
2020, Instituto Mexicano de Tecnología del Agua

Open Access bajo la licencia CC BY-NC-SA 4.0

(https://creativecommons.org/licenses/by-nc-sa/4.0/)

https://larepublica.pe/archivo/839108-la-caida-de-un-gigante-elnevado-huaytapallana/

López-Moreno, J. I., Fontaneda, S., Bazo, J., Revuelto, J., Azorin-Molina, C., Valero-Garcés, B., Morán-Tejeda, E., Vicente-Serrano, S. M., Zubieta, R., \& Alejo-Cochachín, J. (2014). Recent glacier retreat and climate trends in Cordillera Huaytapallana, Peru. Global and planetary change, 1-11. DOI:

https://doi.org/10.1016/j.gloplacha.2013.10.010

Ministerio del Ambiente del Perú. (2016). Tercera Comunicación Nacional del Perú a la Convención Marco de las Naciones Unidas sobre el Cambio Climático. Lima: Ministerio del Ambiente del Perú. Recuperado de http://www.minam.gob.pe/wpcontent/uploads/2016/05/Tercera-Comunicaci\%C3\%B3n.pdf

ONU, Organización de las Naciones Unidas. (12 de noviembre, 1997). Protocolo de Kioto de la Convención Marco de las Naciones Unidas sobre el Cambio Climático. Recuperado de https://unfccc.int/resource/docs/convkp/kpspan.pdf

Panel Intergubernamental del Cambio Climático. (2014). Cambio climático 2014, informe de síntesis. Ginebra, Suiza: Organización Meteorológica Mundial. Recuperado de https://www.ipcc.ch/site/assets/uploads/2018/02/SYR_AR5_FINA L_full_es.pdf

Panel Intergubernamental del Cambio Climático. (2007). Cambio Climático 2007, Informe de Síntesis. Contribución de los Grupos de trabajo I, II y III al Cuarto Informe de evaluación del Grupo 
Tecnología y

Ciencias
2020, Instituto Mexicano de Tecnología del Agua

Open Access bajo la licencia CC BY-NC-SA 4.0

(https://creativecommons.org/licenses/by-nc-sa/4.0/)

Intergubernamental de Expertos sobre el Cambio Climático.

Ginebra, Suiza: IPCC. Obtenido de

https://www.ipcc.ch/site/assets/uploads/2018/02/ar4_syr_sp.pdf

Panel Intergubernamental del Cambio Climático. (2014). Cambio

climático 2014: Impactos, adaptación y vulnerabilidad.

Resúmenes, preguntas frecuentes y recuadros multicapítulos.

Contribución del Grupo de Trabajo II al Quinto Informe de

Evaluación del Grupo Intergubernamental de Expertos sobre

Cambio Climático. Ginebra: Organización Meteorológica Mundial.

Obtenido de

https://www.ipcc.ch/site/assets/uploads/2018/03/WGIIAR5-

IntegrationBrochure_es-1.pdf

Schauwecker , S., Rohrer, M., Acuña, D., Cochachin, A., Dávila, L., \&

Frey, H. (2014). Climate trends and glacier retreat in the

Cordillera Blanca, Peru. Global and Planetary Change, 119(1),

DOI: https://doi.org/10.1016/j.gloplacha.2014.05.005

Senamhi, Servicio Nacional de Meteorología e Hidrología del Perú. (20 de 12 de 2016). Senamhi. Recuperado de https://www.senamhi.gob.pe/?\&p=solicitud-servicio

Vuille, M., Kaser, G., \& Juen, I. (2008). Glacier mass balance variability in the Cordillera Blanca, Peru and its relationship with climate and the large-scale circulation. Global and Planetary Change, (64), DOI: https://doi.org/10.1016/j.gloplacha.2007.11.003

Wikipedia. (2 de noviembre, 2016). Provincia de Huancayo. Recuperado de https://es.wikipedia.org/wiki/Provincia_de_Huancayo 
Zubieta, R., \& Lagos, P. (2010). Cambios de la superficie glaciar en la cordillera Huaytapallana: periodo 1976 - 2006. En: IGP, Cambio climático en la cuenca del río Mantaro (p. 260). Lima, Perú: Ministerio del Ambiente del Perú. Recuperado de http://www.met.igp.gob.pe/publicaciones/2010/libroCC.pdf 